\title{
Effect of Bulk Tissue Motion on Quantitative Perfusion and Diffusion Magnetic Resonance Imaging*
}

\author{
Thomas L. Chenevert and James G. PiPe \\ University of Michigan Medical Center, Department of Radiology, 1500 East Medical Center Drive, \\ Ann Arbor, Michigan 48109
}

Received February 1, 1991; revised February 26, 1991

\begin{abstract}
The effect of irreproducible bulk tissue motions upon quantification of tissue perfusion and diffusion was studied via computer simulation of random phase error in conventional phase-encoded perfusion/diffusion MRI. Simulations using acquisition parameters typical for human brain studies demonstrate that bulk motion irreproducibility of $\approx 60 \mu \mathrm{m} / \mathrm{s}$ can produce phase instability on the order of $20^{\circ}$ which overwhelms estimates of perfusion fraction and produces significant errors in diffusion values. Bulk tissue motion control of the human brain via cardiac gating and substantial head restraint was studied by direct measurement of voxel phase stability. Phase instability of $10^{\circ}$ to $20^{\circ}$ was observed from right-to-left and anterior-to-posterior motions and significantly greater phase variability from superior-to-inferior motion. The spatial pattern of phase variability indicates the source is likely a mixture of cardiac pulsation and respiration. (c) 1991 Academic Press, Inc.
\end{abstract}

\section{INTRODUCTION}

The ability of magnetic resonance to accurately determine diffusion coefficients of isolated samples using the pulsed field gradients is well established $(1,2)$. Successful extension of diffusion measurement methods to the in vivo situation via MRI (3-5), however, is not straightforward due to extraneous semicoherent bulk tissue motions that may overwhelm microscopic incoherent motion effects. The quality of results depend heavily on adequate bulk tissue motion control and system performance. Presence of ghost artifact, whether related to system instability or patient motion, is evidence of signal loss unrelated to the targeted incoherent motions. To date, most investigators have employed cardiac gating to reduce the major source of random phase error (6). Echo-planar imaging (EPI) methods remove random motion phase errors by collecting all phase encoding views with the object in a single motion state (7); however, EPI currently requires specialized hardware. An alternative is to randomize spurious phase effects via signal averaging of time-domain or reconstructed image intensities $(8)$. This approach may lessen gross artifacts by homogenizing errors but does not prevent undesired signal attenuation. Consequently, the true nature of signal loss, and the degree to which coherent bulk motion or system instabilities contribute to the observed signal attenuation, is unknown. Gradient moment nulling

* Presented at SMRM Workshop of Future Directions in MRI of Diffusion and Microcirculation, Bethesda, MD, June 7 and 8, 1990. 
theoretically allows one to eliminate bulk motion phase shifts up to an arbitrary order (9), however, there are severe practical limits since sensitivity to diffusion effects is significantly reduced. For finite gradient amplitude and TE restrictions, examples of standard position-nulled, velocity-nulled, and acceleration-nulled waveforms have relative diffusion sensitivity $1,1 / 4 \mathrm{th}$, and $1 / 18 \mathrm{th}$, respectively. Measurement of incoherent motion relatively free of bulk motion artifact is possible using orthogonal slice-selective pulses, frequency encoding (only), and phase-insensitive signal averaging (10).

The objective of this work is to document bulk motion properties of the human brain with the intent of modeling perfusion/diffusion quantification errors. One data acquisition/processing scenario is studied in detail: cardiac-gated brain imaging, 2D FT spin-echo acquisition at four motion sensitivities, and pixel-by-pixel calculation of perfusion fraction and diffusion using an intravoxel incoherent motion model (4).

\section{METHODS}

Rectangular gradient pulses of amplitude $A$, duration $\delta$, and separation $\Delta$ provide diffusion sensitivity ( 1 ) defined by a $b$ factor given as

$$
b=(\gamma \delta A)^{2}\{\Delta-\delta / 3\} .
$$

Tissue diffusion coefficients are on the order of $D=10^{-3} \mathrm{~mm}^{2} / \mathrm{s}(11)$; therefore, relatively high gradient factors $\left(b \geqslant 10^{2} \mathrm{~s} / \mathrm{mm}^{2}\right)$ are required for adequate diffusion attenuation. Accompanying the high gradient factor is a substantial phase shift per unit velocity when bulk tissue motion is present:

$$
\Phi_{\mathrm{v}}=\gamma A \delta \Delta .
$$

Consider, for example, a spin-echo experiment where $A=10 \mathrm{mT} / \mathrm{m}, \delta=40 \mathrm{~ms}$, and $\Delta=50 \mathrm{~ms}$ ( $\mathrm{TE} \geqslant 90 \mathrm{~ms}$ ). Diffusion attenuation is significant at $\exp (-b D)=0.66$ ( $D=10^{-3} \mathrm{~mm}^{2} / \mathrm{s}$ ); however, even with bulk motion reproducible to $\pm 100 \mu \mathrm{m} / \mathrm{s}$ the phase instability is still poor $\left( \pm 30^{\circ}\right)$.

\section{Simulation of Motion Artifact in Perfusion/Diffusion Estimates}

The effect of motion phase error was studied using a one-dimensional phase-encoded object model. Let $S_{A}(0)$ represent the central reconstructed pixel of a data set acquired with diffusion gradient amplitude $=A$,

$$
S_{A}(0)=\alpha_{A} \int S_{o}\left(k_{y}\right) e^{i \Phi_{v} v\left(k_{y}\right)} d k_{y} .
$$

The ideal attenuation, $\alpha_{A}$, is due to perfusion and diffusion effects, $S_{o}\left(k_{y}\right)$ is the Fourier transform of the unattenuated object, and the phase shift results from undesired rigid body motion at speed $v\left(k_{y}\right)$ and varies with each phase encoding step $k_{y}$. Uniform perfusion and diffusion properties are implicit in Eq. [3]. This model was used in computer simulation of random bulk motion phase errors. In order to focus on motion artifacts, other aspects of the object were simplified. The 128-point phase-encoded rectangular object was noiseless and had uniform isotropic perfusion (perfusion volume fraction $=5 \%)$ and diffusion $\left(D=100 \times 10^{-5} \mathrm{~mm}^{2} / \mathrm{s}\right)$. Random bulk motion (linear 
speed only) was normally distributed with zero mean. Gradient factors were modeled as: $\delta=36 \mathrm{~ms} ; \Delta=63 \mathrm{~ms} ; A=0,4.6,6.9,9.5 \mathrm{mT} / \mathrm{m}$. A three-point linear fit to the logarithm ratio, $\ln \left\{\left|S_{0}\right| /\left|S_{A}\right|\right\}$, as a function of $b$ factor yields slope and intercept for estimation of diffusion and perfusion fraction, respectively. Repeated runs $(N=256)$ for a given standard deviation of bulk motion speed allowed calculation of the reproducibility of perfusion and diffusion estimates in the presence of random phase.

\section{Measurement of Bulk Tissue Motion Phase Shifts in Vivo}

Phase stability of voxels localized in the human brain was measured directly to augment the simulations. Acquisitions were cardiac gated $(T R \approx 2 \times E C G \approx 1.8 \mathrm{~s}$ ) at trigger delays of 100,350 , and $500 \mathrm{~ms}$. Orthogonal $90^{\circ}$ and $180^{\circ}$ slice-selective pulses confined sensitivity to a $10 \times 10$-mm right-to-left oriented column in the brain of a normal cooperative volunteer. The column was located through the frontal lobes and anterior horn of lateral ventricles just anterior to the foramen of Monroe. The head was immobilized using a vacuum bag (Vac-Pac Size 20, Olympic Medical, Seattle, WA) backed by Velcro fixed to the head coil. Air bags inflated in place partially filled the residual space within the head coil for improved immobilization. Frequency encoding along the column axis provided one-dimensional localization from which echoto-echo phase stability of individual voxels could be quantified directly. Forty-one echoes for each motion-sensitive gradient direction $(\mathrm{R} / \mathrm{L}, \mathrm{S} / \mathrm{I}$, and $\mathrm{A} / \mathrm{P})$ at amplitudes of 0 and $9 \mathrm{mT} / \mathrm{m}(\delta=36 \mathrm{~ms}, \Delta=66 \mathrm{~ms})$ were sampled iteratively in a single scan (total scan time $\approx 8 \mathrm{~min}$ ). In addition, a static phantom was scanned to provide a phase reference such that average phase shift could be calculated.

\section{RESULTS AND DISCUSSION}

Simulations were performed for a range of bulk motion conditions defined by the resultant standard deviation of phase. Results are summarized in Fig. 1 as the mean
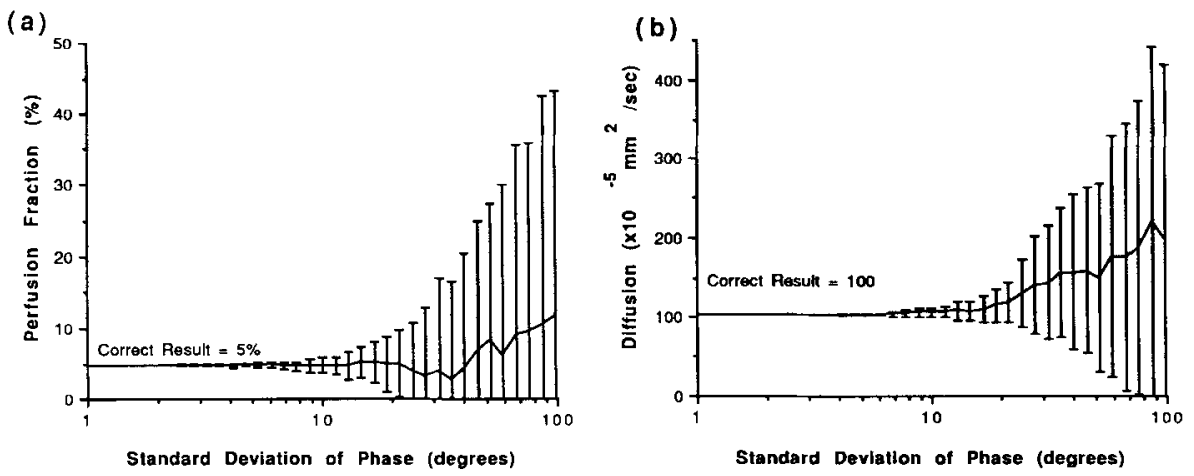

FrG. 1. Simulation of calculated (a) perfusion fraction and (b) diffusion in the presence of random phase. The mean and standard deviation (error bars) of 256 calculations are displayed as a function of phase variability. The phase variability corresponds to the highest gradient setting of four used to calculate perfusion and diffusion using an IVIM model. 


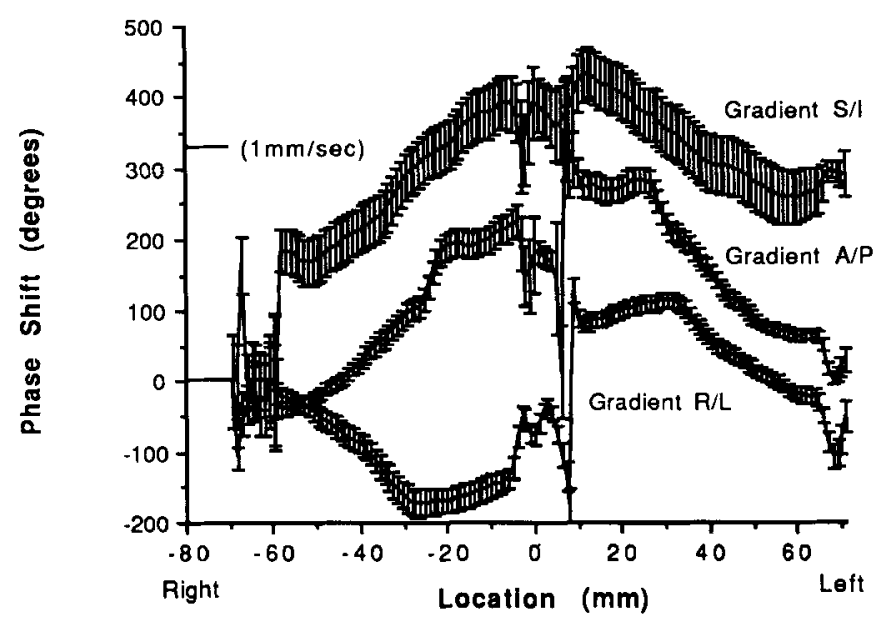

FIG. 2. Temporal mean and standard deviation (shown as error bars) of phase due to motion along $\mathrm{S} / \mathrm{I}$, $\mathrm{A} / \mathrm{P}$, and $\mathrm{R} / \mathrm{L}$ directions. The $10 \times 10-\mathrm{mm}$ tissue column was oriented $\mathrm{R} / \mathrm{L}$ through the brain. Nominal conversion from phase shift to linear speed is $325^{\circ} / \mathrm{mm} / \mathrm{s}$.

and standard deviation of the calculated perfusion fraction and diffusion from 256 simulated runs as a function random phase severity. Within limits where the simulation is valid, it is apparent that relatively minor motion instability has a pronounced impact particularly on calculated perfusion fraction. For example, a $20^{\circ}$ standard deviation in phase (bulk motion controlled to $\pm 60 \mu \mathrm{m} / \mathrm{s}$ ) produces $\approx 5 \%$ variability in perfusion fraction which represent a $100 \%$ relative error (known perfusion fraction value is $5 \%$ ).

The temporal average and standard deviation in phase along the column in the brain is shown in Fig. 2 for an ECG delay of $100 \mathrm{~ms}$. Absolute tissue speed is difficult to quantify since the motion encoding period spans $\approx 100 \mathrm{~ms}$ and there may be errors in phase unwrapping, particularly for CSF motion in the ventricles; nevertheless, tissue
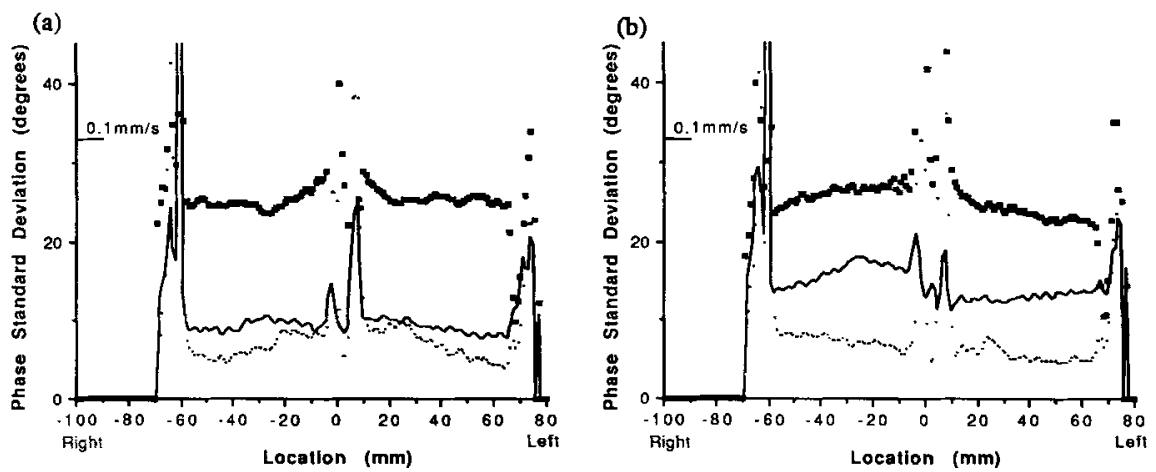

FIG. 3. Temporal standard deviation of phase (measured over 41 echoes) due to motion along $S / I(\square)$, $\mathrm{A} / \mathrm{P}(\cdots)$, and $\mathrm{R} / \mathrm{L}(-)$ directions. The $10 \times 10$-mm tissue column was oriented $\mathrm{R} / \mathrm{L}$ through the brain. Cardiac-gated acquisitions were at (a) $350 \mathrm{~ms}$ ECG delay and (b) $500 \mathrm{~ms}$ ECG delay. 
speeds can exceed $1 \mathrm{~mm} / \mathrm{s}$. The greatest speed is in the $S / I$ direction in agreement with observations of others (12). Relatively high tissue speed is not the source of perfusion/diffusion quantification errors, but rather irreproducibility of such speed. Phase variability due to bulk motion variability in the human brain is illustrated in Figs. $3 \mathrm{a}$ and $3 \mathrm{~b}$ for ECG delays of 350 and $500 \mathrm{~ms}$, respectively. Clearly the greatest motion variability is in the $\mathrm{S} / \mathrm{I}$ direction and is on the order of $100 \mu \mathrm{m} / \mathrm{s}$ resulting in a random phase of $\approx 30^{\circ}$ for this individual and these experimental conditions. Other motion directions allowed phase reproducibility to within $20^{\circ}$ in the solid brain tissue. A similar experiment on another volunteer yielded significantly poorer motion control indicating intersubject variability is yet another consideration.

The spatial pattern of phase instability supports the notion both cardiac and respiration motions are irreproducible, although the relative contributions are unknown. The magnitude of phase instability demonstrated in Fig. 3 and reference to the simulation results indicate substantial motion artifact corruption of perfusion/diffusion values can be expected if conventional cardiac-gated phase-encoded acquisition / processing methods similar to those modeled are used.

\section{REFERENCES}

1. E. O. STEJSkal And J. E. TANner, J. Chem. Phys. 42(1), 288 (1961).

2. J. E. TANNER, Biophys. J. 28(1), 107 (1979).

3. D. LE BIHAN, Magn. Reson. Med. 7, 346 (1988).

4. D. Le Bihan, E. Breton, D. Lallemand, M. L. Aubin, J. Vignaud, and M. Laval-Jeantet, Radiology 168(2), 497 (1988).

5. K. D. MERboldt, W. Hanicke, ANd J. FrahM, J. Magn. Reson. 64, 479 (1985),

6. K. D. Merboldt, W. Hanicke, M. L. Gyngell, J. Frahm, and H. Bruhn, Magn. Reson. Med. 12, 198 (1989).

7. R. Turner, D. Le Bihan, J. Maier, R. Vavrex, L. Kyle Hedges, And J. Pek ar, Radiology 177, 407 (1990).

8. K. D. Merboldt, H. Bruhn, J. Frahm, M. L. Gyngell, W. Hanicke, and M. Deimling, Magn. Reson. Med. 9, 423 (1989).

9. N. Fujita, K. Harada, K. Sakurai, T. Murakami, S. Kim, Y. AKai, and T. Kozuka, in "Book of Abstracts, Society of Magnetic Resonance" 907, 1989.

10. T. L. Chenevert, J. G. Pipe, D. M. Williams, ANd J. A. Brunberg, Magn. Reson. Med. 17, 197 (1991).

11. R. L. CoOper, D. B. Chang, A. C. Young, C. J. Martin, And D. AnCKer-Johnson, Exp. Biophys. J. 14(3): 161 (1974).

12. D. A. FeINBERG AND A. S. MARK, Radiology 163(3), 793 (1987). 